\title{
Simulation of gas kicks during oil well drilling
}

\author{
ROLV ROMMETVEIT $\uparrow$ and ANETTE BLYBERG†
}

Keywords: Computer simulator, gas kicks, water-based versus oil-based mud kicks.

A computer simulator is needed to calculate expected development of gas kicks. Such a simulator is presented, and some simulation examples are shown. The model is flexible, and can be used for research studies, well planning and training purposes. Several simulation examples are shown which illustrate some of the differences between kicks in WBM and OBM.

\section{Introduction}

The work to prevent and control gas kicks is taken seriously by both the authorities and the oil industry. Even if most kicks are brought under control, a blowout can result in grave damage to life and the environment and economic losses of millions of pounds.

To describe and analyse kick phenomena, computer models have been developed. Previously these models were based on over-simplified assumptions, for instance about the gas distribution in the well annulus. This resulted in erronous predictions. Today more advanced models have been developed, which simulate the dynamic response from the well on a kick in water-based drilling mud rather well (Ekrann and Rommetveit 1985, Nickens 1985). For kicks in oil-based muds only two dynamic models have been published (Ekrann and Rommetveit 1985, Swanson, Gilvary and McEwan 1988).

A kick in oil-based drilling mud is more difficult to detect due to the considerable gas solubility in the base oil at high pressures. Accordingly, well control related questions should be treated with special thoroughness while drilling with oil-based mud in areas with gas. This paper presents the GASKICK computer model, and results from simulations with the simulator are also given.

\section{Gas kicks}

2.1. Reasons for gas kicks

A gas kick is said to occur when gas passes into the wellbore. A gas kick may arise for a variety of reasons,

(i) Too low mud density

(ii) Piston effect while tripping

(iii) Low fluid level in the annulus, due to lost circulation or incomplete filling up of hole while pulling out

(iv) Drilling through porous rocks or high-pressure zones

(v) Diffusion of gas through the mud cake

Received 1 August 1989.

$\dagger$ Rogaland Research Institute, P.O. Box 2503 Ullandhaug, 4004 Stavanger, Norway.

This paper was presented at SIMS '89 (Scandinavian Simulation Society), 31st Annual Meeting, Bergen, Norway, May 31--June 2, 1989. 


\subsection{Detection of gas kicks}

When no downhole measurements are available for predicting abnormally pressured formations or detecting inflow of gas into the well, one has to rely on monitoring the mud flow and interpreting flow parameters.

The following conditions may indicate a kick,

(i) Increase in the rate of penetration

(ii) A decrease in pump pressure

(iii) Improper hole fill while tripping out

(iv) Increased flow in flowline

(v) Mud pit gain

(vi) Gas cut mud

(vii) Return flow with pump off

\subsection{Controlling a gas kick}

The basic approach in kick killing is to shut the well in by means of the BOP, and eventually circulate the kick out by pumping weighted mud into the system through the kill-line. The gas is circulated out through the choke-line.

\section{The kick process}

The development of a gas kick from the start of inflow until the gas is circulated out through the choke-manifold, is a complex interaction of many different subprocesses. These subprocesses interact and external factors such as well geometry, mud and gas properties, reservoir conditions, time of detection, control procedures etc., determine the character of the kick.

A gas kick in oil-based mud will be more complex than in water-based mud due to the high solubility of the gases in the base oil of the mud. As long as the gas is dissolved no gas expansion is experienced, so the influx is 'hidden' in oil-based compared to water-based mud. When the gas flashes out of the mud, the rest of the kick will develop more rapidly since both gas flashing and free gas expansion take place simultaneously.

The following parameters are important for the development of a gas kick in oil based mud:

MUD PROPERTIES

GAS

WELL/RESERVOIR

OPERATIONS

FACTORS
Mud density

Base oil type

Volumetric content of base oil

Gas type

Gas solubility

Well geometry (depth, deviation, capacity)

Reservoir properties

Temperature gradient

Pump flowrate

Drilling velocity (ROP)

Time of detection

Control procedures 
It is almost impossible to consider all these factors by conventional calculations. A dynamic computer model has been developed for the process (Ekrann and Rommetveit 1985). This model accounts for all important factors during a kick in oil based mud, and will be verified with full-scale experimental data recorded under controlled conditions (RRI, Stavanger 1988).

\section{Overview of physical system}

The geometry of the real system is sketched in Fig. 1. Mud is pumped down the drill string, through the drill bit and up the annulus. This constitutes a flow line. The geometry of the flow line can be changed by closing the BOP and thus diverting the flow through the choke. Gas can enter the annulus from a reservoir, being carried upwards and out of the system either at the top of the annulus, or through the choke, as the case may be.

Of primary importance is the dynamics of a gas kick. The fluid flow is approximated as being 1-dimensional. Thus, the independent variables of the mathematical problem are time and one spatial coordinate (i.e. length along flow line). The computational domain extends from the top of the drill string to the end of annulus or choke. As boundary conditions we use some pump model at the inlet, and assume atmospheric pressure at the outlets.

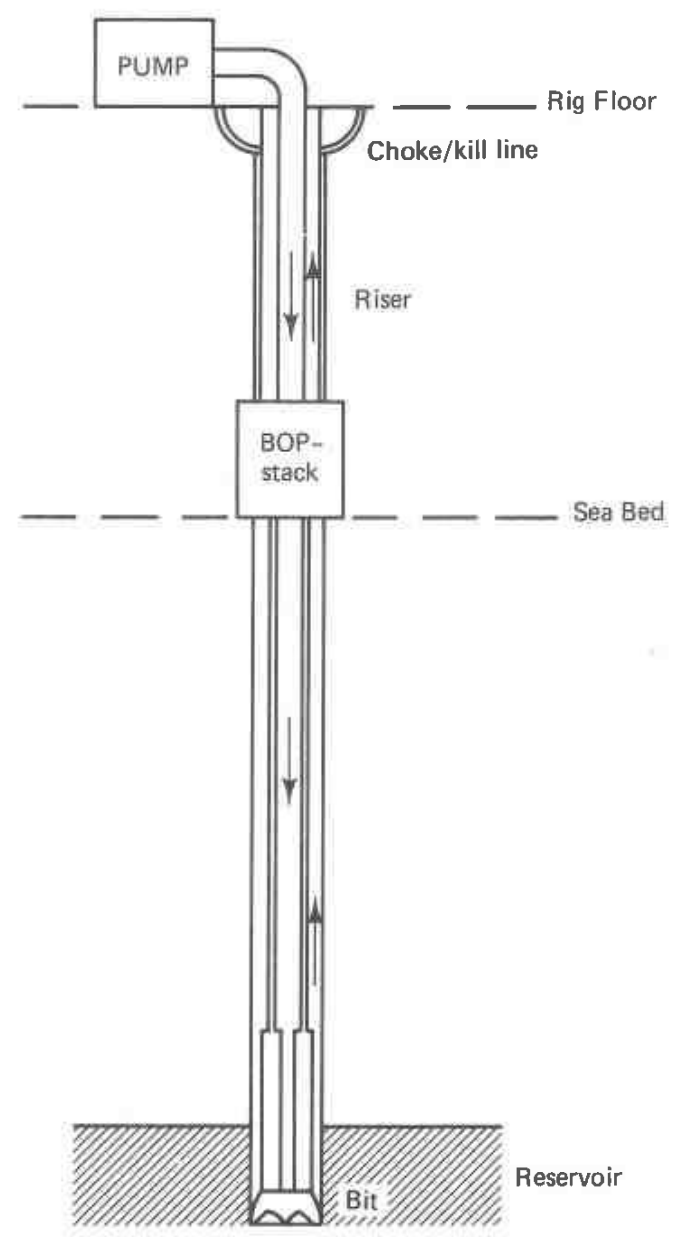

Figure 1. Schematic outline of the real physical system. 
The main dependent variables of the problem are pressure, fluid velocities (liquid and free gas), and mass/volume of mud, dissolved gas and free gas. Thus, these variables are computed as a function of time and position along flow line.

Temperature is assumed known. Thus, the governing equations are basically those expressing conservation of mass and momentum.

The choice of the geometry of the system (angles, cross sections, lengths) is free, but remains fixed during a computation. This implies that drilling is not simulated. However, height of the reservoir can be made variable with time, to allow for the main effect of drilling.

During a simulation, the following parameters can be interactively changed: BOP status (closed/open), choke setting, pump power, type of mud pumped.

The following physical effects are included in the model

Frictional pressure losses, both for one phase and two phase flow

Pressure loss in bit

Pressure loss in choke

Viscosity variations with pressure, temperature and composition

Density variations with pressure, temperature and gas content

Dissolution of gas in mud

Dynamics of dissolution of gas in mud (non-equilibrium)

Rise velocity of gas

Reservoir dynamics

\section{Mathematical model}

We assume that all variables depend on only one spatial coordinate (length along flow line) and neglecting effects from cross sectionally non-uniform velocity profiles and mass distribution profiles. The temperature is also assumed to be known at each point along the flow line. The fluid system is treated as a black oil system. Gas can exist either as free gas or dissolved gas.

The basic equations are

Mass conservation of mud

$$
\frac{\partial}{\partial t}\left(A(1-\alpha) \rho_{1}\right)=-\frac{\partial}{\partial s}\left(A(1-\alpha) \rho_{1} v_{1}\right)+A \dot{m}_{\mathrm{g}}
$$

Mass conservation of free gas

$$
\frac{\partial}{\partial t}\left(A \alpha \rho_{\mathrm{g}}\right)=-\frac{\partial}{\partial s}\left(A \alpha \rho_{\mathrm{g}} v_{\mathrm{g}}\right)-A \dot{m}_{\mathrm{g}}+q
$$

Mass conservation of dissolved gas

$$
\frac{\partial}{\partial t}\left(A(1-\alpha) \rho_{1} x\right)=-\frac{\partial}{\partial s}\left(A(1-\alpha) \rho_{1} x v_{1}\right)+A \dot{m}_{\mathrm{g}}
$$

Conservation of total momentum

$$
\begin{aligned}
\frac{\partial}{\partial t}\left((1-\alpha) \rho_{1} v_{1}+\alpha \rho_{\mathrm{g}} v_{\mathrm{g}}\right)= & -\frac{\partial p}{\partial s}-F+\left((1-\alpha) \rho_{1}+\alpha \rho_{\mathrm{g}}\right) g \cos \theta \\
& -\frac{\partial}{\partial s}\left((1-\alpha) \rho_{1} v_{1}^{2}+\alpha \rho_{\mathrm{g}} v_{\mathrm{g}}^{2}\right)
\end{aligned}
$$


The above constitutes 4 equations for 10 unknowns $\left(\alpha, \rho_{1}, x, v_{1}, \rho_{\mathrm{g}}, v_{\mathrm{g}}, q, \dot{m}_{\mathrm{g}}, p, F\right)$. Also, we propose to keep track of the different muds pumped into the system, giving still more unknowns.

To close the system, we need six more equations. These equations are normally algebraic equations. For each equation there are several alternatives. The equations are referred to as submodels. The functional relationships are

Gas density

$$
\rho_{\mathrm{g}}=\rho_{\mathrm{g}}(p, T)
$$

Mud density

$$
\rho_{1}=\rho_{1}(p, T, n, x)
$$

Free gas velocity

$$
v_{\mathrm{g}}=v_{\mathrm{g}}\left(p, T, n, x, \alpha, s, v_{1}, v_{\mathrm{s}}\right)
$$

Gas influx

$$
q=q(p, s, t)
$$

Rate of gas dissolution

$$
\dot{m}_{\mathrm{g}}=\dot{m}_{\mathrm{g}}\left(p, T, x, \alpha, v_{1}, v_{\mathrm{g}}, s\right)
$$

Frictional pressure loss

$$
F=F\left(p, T, n, \alpha, v_{1}, v_{\mathrm{g}}, s\right)
$$

The symbols used above are

$t$ time

$s$ length coordinate

$A(s)$ flow line cross sectional area

$\alpha$ volume fraction of free gas

$\rho_{1}$ mud density

$\rho_{\mathrm{g}}$ gas density

$x$ mass fraction of dissolved gas in mud

$v_{1}$ flow velocity of mud

$v_{\mathrm{g}}$ flow velocity of gas

$v_{\mathrm{s}}$ gas slip velocity

$q$ gas inflow rate from reservoir

$\dot{m}_{\mathrm{g}}$ rate of dissolution of free gas

$p$ pressure

$F$ pressure loss (frictional, in bit, choke, and changes of flow area)

$g$ acceleration of gravity

$\theta$ flow line angle with vertical

$T(s)$ temperature

$n(s, t)$ mud number

There is one basic approximation to be made before our system of equations is solved numerically. We delete the time derivative term and the momentum transport term in 
eqn. (4). This will exclude pressure propagation effects from the equations, and provide for a quasi-stationary approximation. Equation (4) now reads

$$
\frac{\partial p}{\partial s}+F=\left((1-\alpha) \rho_{1}+\alpha \rho_{\mathrm{g}}\right) g \cos \theta
$$

A finite difference predictor corrector shooting method is employed for the solution of the set of non-linear equations arising at each time step.

In the following the submodels are briefly described.

\section{Mud density}

A model for mud density as a function of dissolved gas content, composition, pressure and temperature has been developed. The main components in the mud are base oil, water and solids. Base oil and water are compressible, and solids are incompressible. The mud density can be varied interactively by changing the weight material content.

The water phase density at variable pressure and temperature is calculated by correlations. The base oil density can alternatively be computed by means of PVT-data from tables. Dissolved gas is considered as a part of the base oil, and the base oil density as a function of dissolved gas concentration is computed either by means of correlations or tabular values.

2. Gas density

The real gas law is used to calculate $\rho_{\mathrm{g}}$ as a function of pressure and temperature. The gas compressibility factor is calculated by means of correlations.

3. Free gas velocity

Both bubble and slug flow regimes can exist during kick. Separate models for slip velocity $v_{s}$ are used in these two regimes.

4. Gas solubility

Determination of the amount of dissolved gas in the base oil at saturation is done either by means of correlations or tabular solubility data.

5. Dynamic gas dissolution

The rate of gas solution is controlled by diffusion. It is a function of in situ dissolved gas concentration, gas concentration at saturation, flow regime and gas bubble size.

6. Rheology model

Both the Bingham and the Power-Law models are implemented in the simulator.

A model for pressure and temperature dependent viscosity of the mud is implemented.

The model also includes thinning effects due to dissolved gas. A model for twophase mixture viscosity is also included in the simulator.

7. Frictional pressure losses

Single phase frictional pressure losses are calculated by means of friction factor correlations developed for non-Newtonian fluids.

Two-phase frictional pressure losses are calculated either by using correlations developed for mud/gas flows, or by modifying correlations developed for Newtonian fluids/gas. 
The modifications are

(i) Use friction factor correlations developed for non-Newtonian fluids.

(ii) Compute equivalent liquid viscosity by means of a non-Newtonian model.

8. Localized pressure loss

Localized pressure losses are modeled in drillbit, choke, chokeline and at flow area changes.

\section{Computer model}

\subsection{Overview}

The computer model itself is named GASKICK. It operates in an environment as illustrated below, where the arrows indicate direction of flow of information. All input concerning geometry, fluid properties, choice of submodels, output commands, etc. is made from the INPUT file.

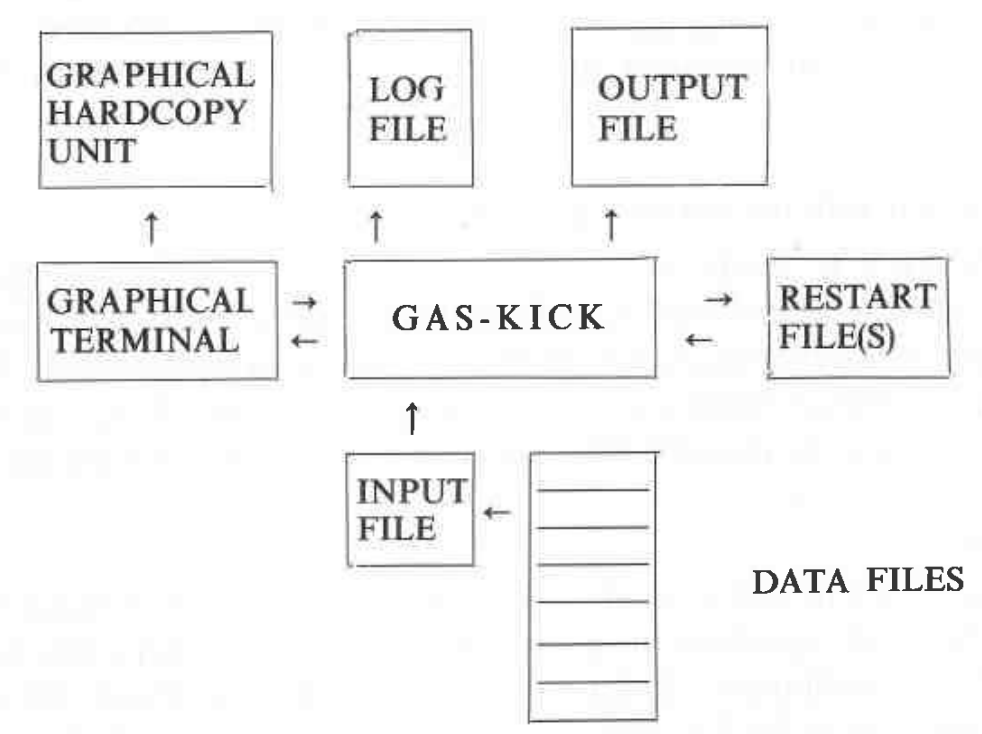

The OUTPUT file, typically a line printer, will be used for writing a copy of the INPUT file, various information on geometry, discretization, fluid properties, submodels, etc., as well as actual computational results, all in table form.

When computations are stopped before a complete simulation has been performed, the RESTART file is used to write information complete enough for a continuation of the simulation in a later run, thus avoiding the need for a recomputation.

A simulation is controlled interactively from a graphical TERMINAL. A HOLD function will permit the computation to be temporarily halted. In HOLD mode, commands may be issued to the simulator. The commands can change BOP, choke and pump status, as well as type of mud pumped, simulating actions which it is possible to take in real life situations.

Information received on the terminal is essentially of two types

(1) Plots of variables (pump pressure, pit volume gain, input flow rate, output flow rates) versus time. This is typical information received in real life situations. 
(2) Plots of variables (pressure, velocities, mass/volume fraction of mud and gas) versus spatial coordinates. After each time step in the simulation, the plots are updated. This is information normally not received in real life.

Also, current status of BOP, choke, pump, etc. will appear in both cases. In HOLD mode, the operator can switch between four screen pictures, illustrating the various parameters.

The graphical TERMINAL will have a HARDCOPY unit attached. In HOLD mode, the operator can obtain hardcopy of the current screen picture. The LOG file logs all terminal transactions, and is intended to function as a debugging help.

\subsection{Modularity}

It is recognized that, for most of the physical effects included in the model, there is no single accepted mathematical description. For many of the effects, there exists a large number of alternative formulations.

Therefore, GASKICK is programmed to be very modular. New alternative submodels can easily be added to the program. Already, for some effects, a large number of alternative submodels have been programmed. The choice of submodels, from the library of programmed ones, can be done via commands in the input file.

\section{Simulations with the GASKICK model}

The GASKICK model has several applications, for research purposes, well planning and field-related studies, and for training purposes. A special training version with advanced, interactive colour graphics is now under development. The simulator treats both gas and oil (with dissolved gas) kicks. The drilling fluid system can be both water or oil based. In the following some simulation examples are presented.

\subsection{A real kick}

A real gas kick in water-based mud has been simulated (Rommetveit and Blyberg (1986)). All relevant data from the kick (well geometry, mud properties, pump rate etc.) were used. The development and control of the kick was simulated. The kick occurred during tripping in a $3612 \mathrm{~m}$ deep well. The pit gain was $9 \mathrm{~m}^{3}$ when the kick was detected, and increased to $17 \mathrm{~m}^{3}$ when the well was closed in.

The simulation is compared to real kick data on Figure 2. The agreement is relatively good.

\subsection{A kick in oil-based mud}

When gas flows into a well during drilling with oil-based mud, the gas will dissolve in the base oil. The maximum dissolved gas concentration is determined by pressure and temperature. When the mud/gas mixture is pumped upwards in the well, the mixture will reach its bubble point, and free gas will flash out from the mud. Hereafter both free gas expansion and additional flashing will occur. If the flashing starts in the riser prior to detection of the kick, the gas will reach the rig area, thereby creating difficult situations.

Figure 3 shows such a simulated kick. The annulus contains dissolved gas in its whole length, and free gas has broken out in the riser. At the point when the flashing started the pit gain was only $0.5 \mathrm{~m}^{3}$, which is below the detection limit of the kick. 


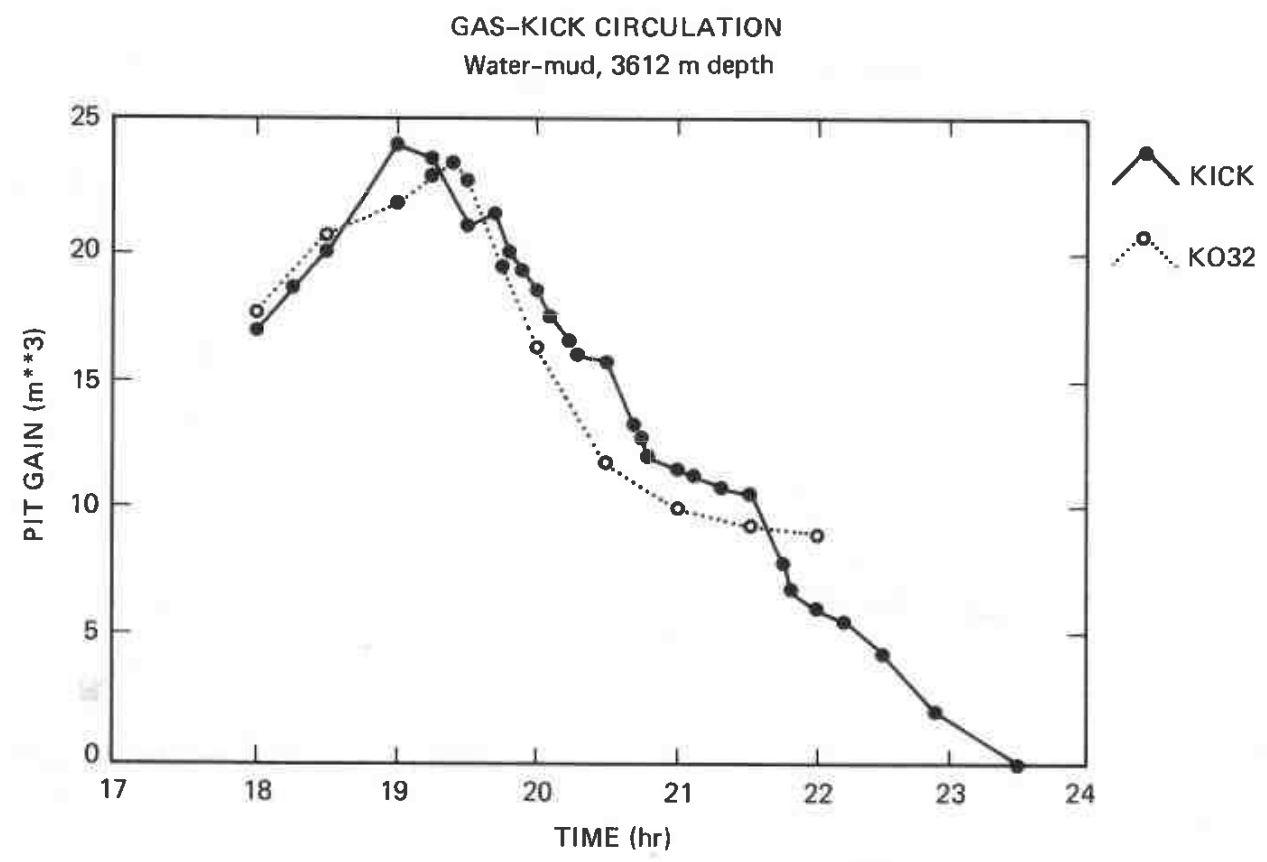

Figure 2. Real kick versus simulated kick. Observed and computed pit gain plotted versus time.
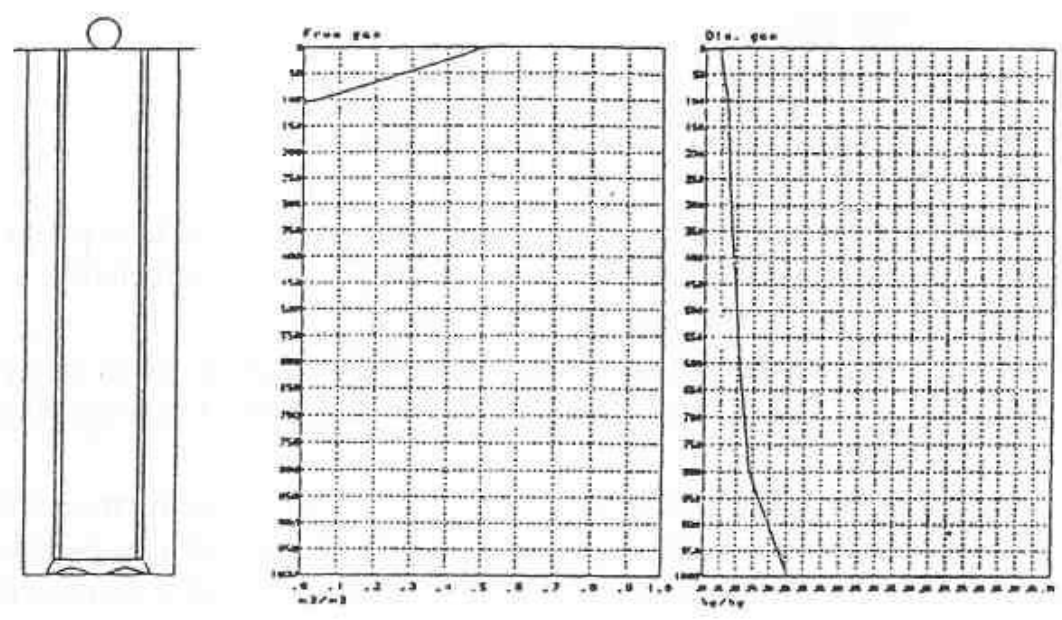

Figure 3. Simulated kick in oil-based mud in a vertical $1000 \mathrm{~m}$ deep well 23,5 minutes after start of gas influx. The right curve shows weight fraction of dissolved gas. The left curve shows that free gas has broken out in the upper $100 \mathrm{~m}$ of the well. 


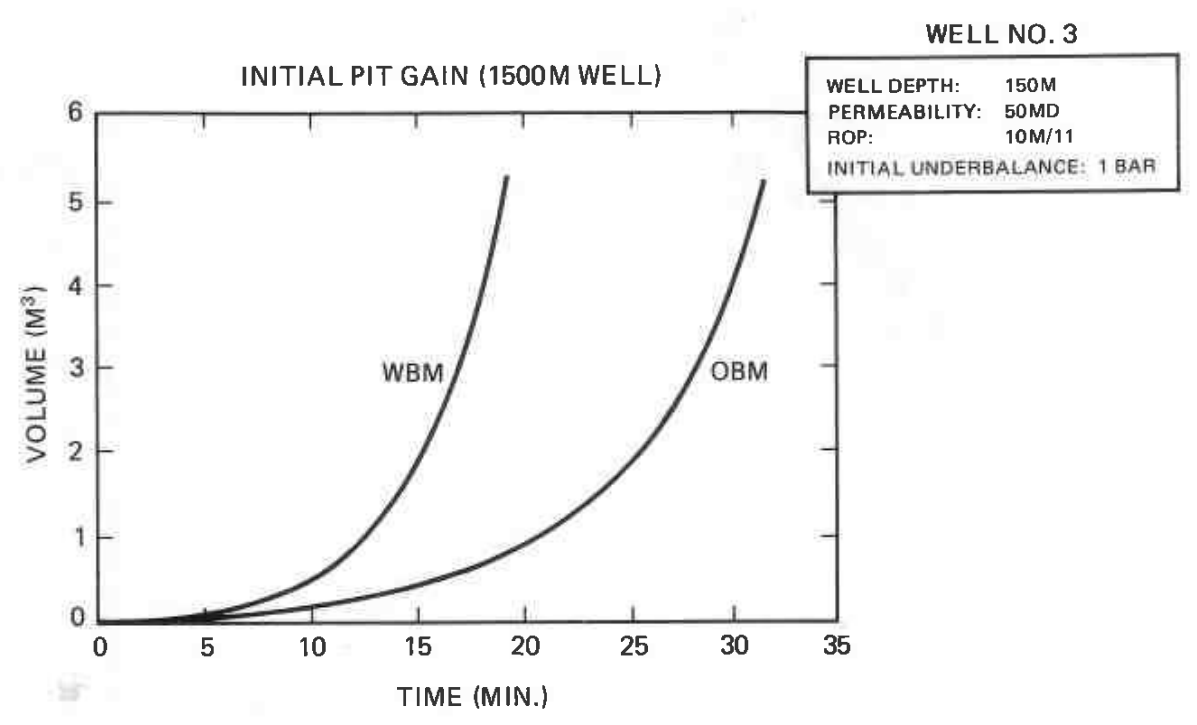

Figure 4. Surface pit gain versus time for a kick in OBM (right curve) and WBM (left curve).

\subsection{Kicks in oil-based versus water-based muds}

There is a wide variety of kicks in OBM. In this section a kick in OBM which occur during drilling will be discussed and compared to a kick in WBM.

\subsubsection{Surface pit gain}

Figure 4 shows surface pit gain versus time for WBM and OBM respectively. The simulation parameters were

$\begin{array}{ll}\text { Well depth } & 1500 \mathrm{~m} \\ \text { Mud density } & 1,43 \mathrm{~kg} / \mathrm{dm}^{3} \\ \text { Pump flow rate } & 20001 / \mathrm{min} \\ \text { Initial formation pressure } & \mathrm{BHP}+1 \mathrm{Bar} \\ \text { Permeability } & 50 \mathrm{mDarcy} \\ \text { Porosity } & 40 \% \\ \text { Rate of penetration } & 10 \mathrm{~m} / \mathrm{hr}\end{array}$

The OBM case gives a much smaller rate of increase of surface pit gain than the WBM case. The consequence of this is that the kick will be detected at a much later stage, and much closer to the surface in the OBM case.

The total amount of gas which has entered the well at the point of detection will always be higher in kicks in OBM. This is due to total volume shrinkage when free gas is dissolved in the mud.

For formations with low permeability and low ROP, or for formations only a few metres thick, the difference between kicks in OBM and WBM can be even larger. In such situations the dissolved gas can break out in the riser prior to detection (see Fig. 3), and this is a potentially dangerous situation.

\subsection{Control phase of kicks in $O B M$}

The surface manifestation of the control phase of a kick in OBM is focused in this section. Figure 5 shows the pit gain versus. time for two kicks in WBM and OBM 


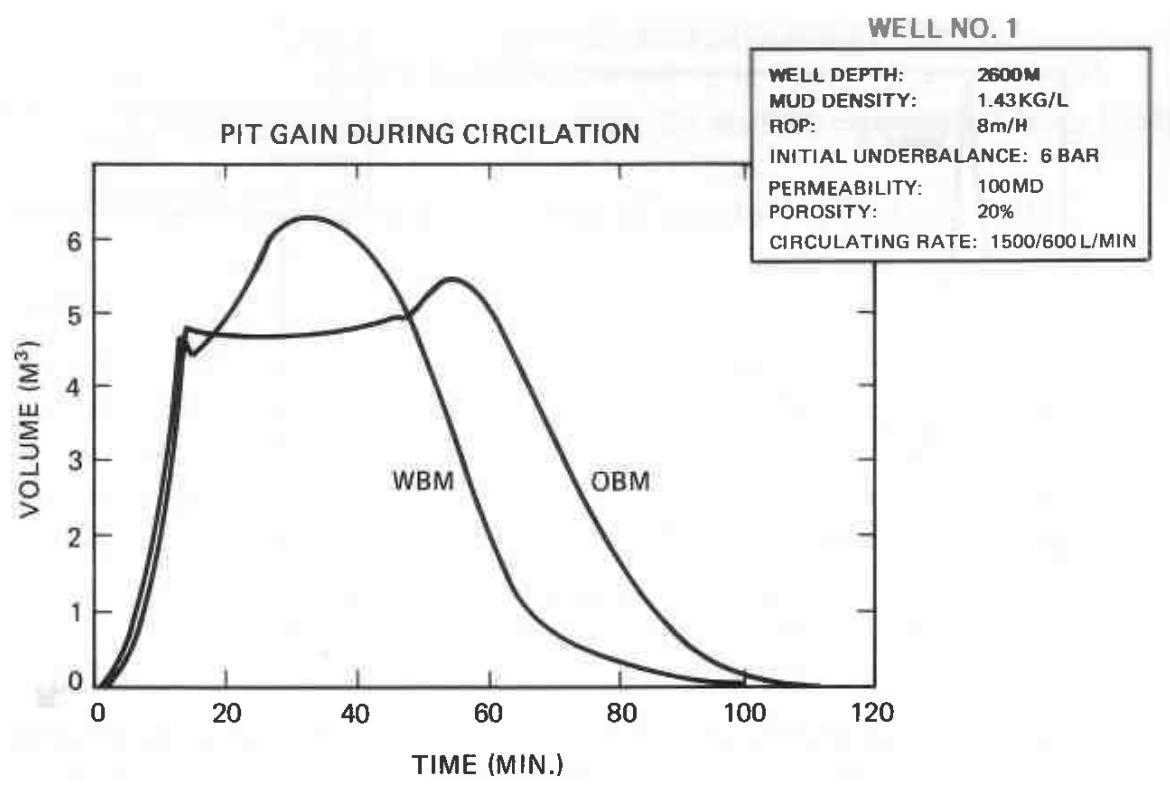

Figure 5. Pit grain versus time for gas kicks in OBM and WBM respectively in a $2600 \mathrm{~m}$ deep well.

respectively with identical initial differential pressures. The well depth in $2600 \mathrm{~m}$. Both kicks are circulated out with the drillers method. The relevant well, reservoir and operational data are also given on Fig. 5. Figure 6 shows gas flow rate through the choke for the two kicks.

On Figs. 7 and 8 pit gain the gas flow rate through the choke versus time are plotted for two kicks in WBM and OBM in a $1500 \mathrm{~m}$ deep well. Comparing the two situations (well no. 1 versus well no. 2) we see that the permeability, underbalance and ROP are

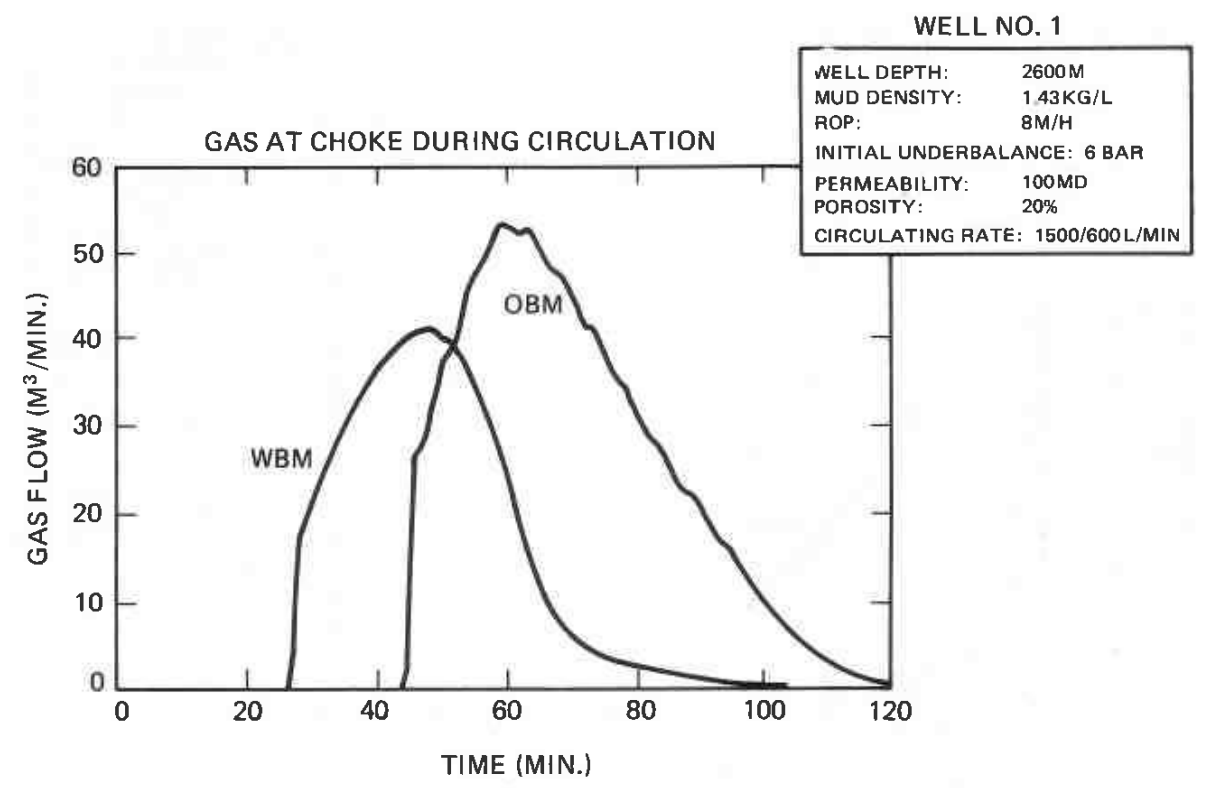

Figure 6. Gas flow rate through choke versus time for the kicks in the $2600 \mathrm{~m}$ deep well. 


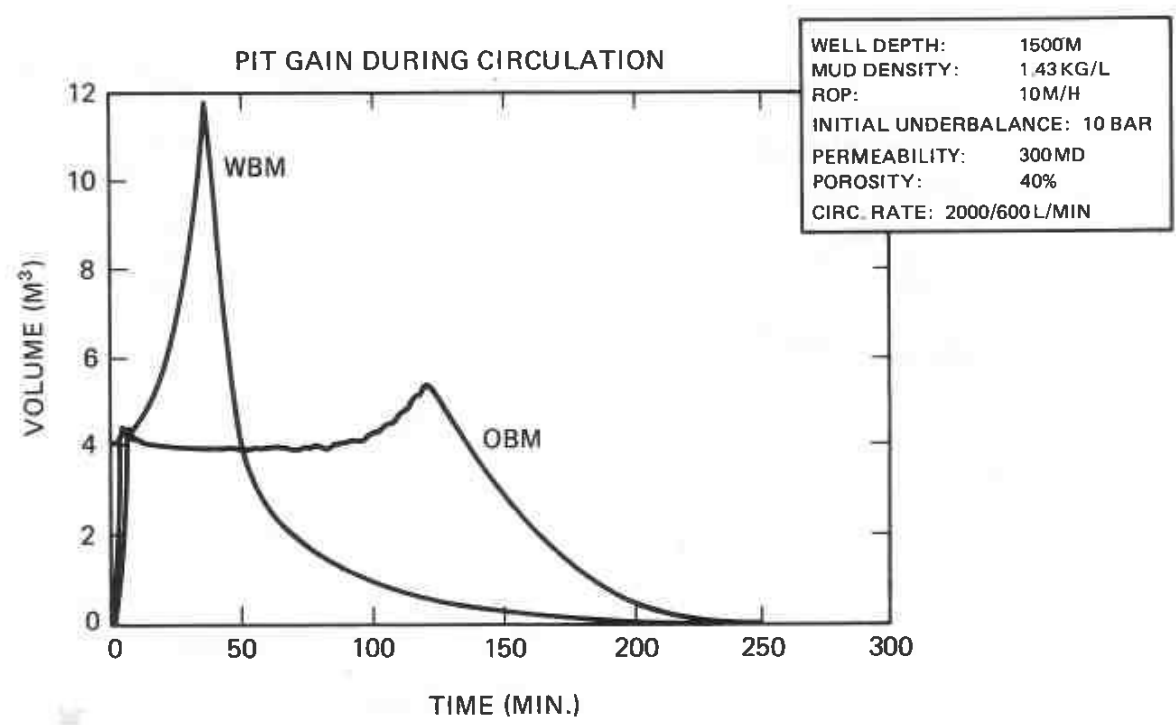

Figure 7. Pit gain versus time for gas kicks in OBM and WBM respectively in a $1500 \mathrm{~m}$ deep well.

highest in well no. 2, thus giving a higher influx rate in this case. The following comments are made

(i) The maximum pit gain will always be less in OBM than in WBM for kicks with identical initial conditions.

(ii) The pit gain will stay constant or decrease in OBM, until the gas approaches the surface and breaks out of solution again. If the initial gas influx is violent so that the mud is saturated with gas and also a lot of free gas exists, the pit gain will decrease after close in and during circulation due to free gas dissolving again.

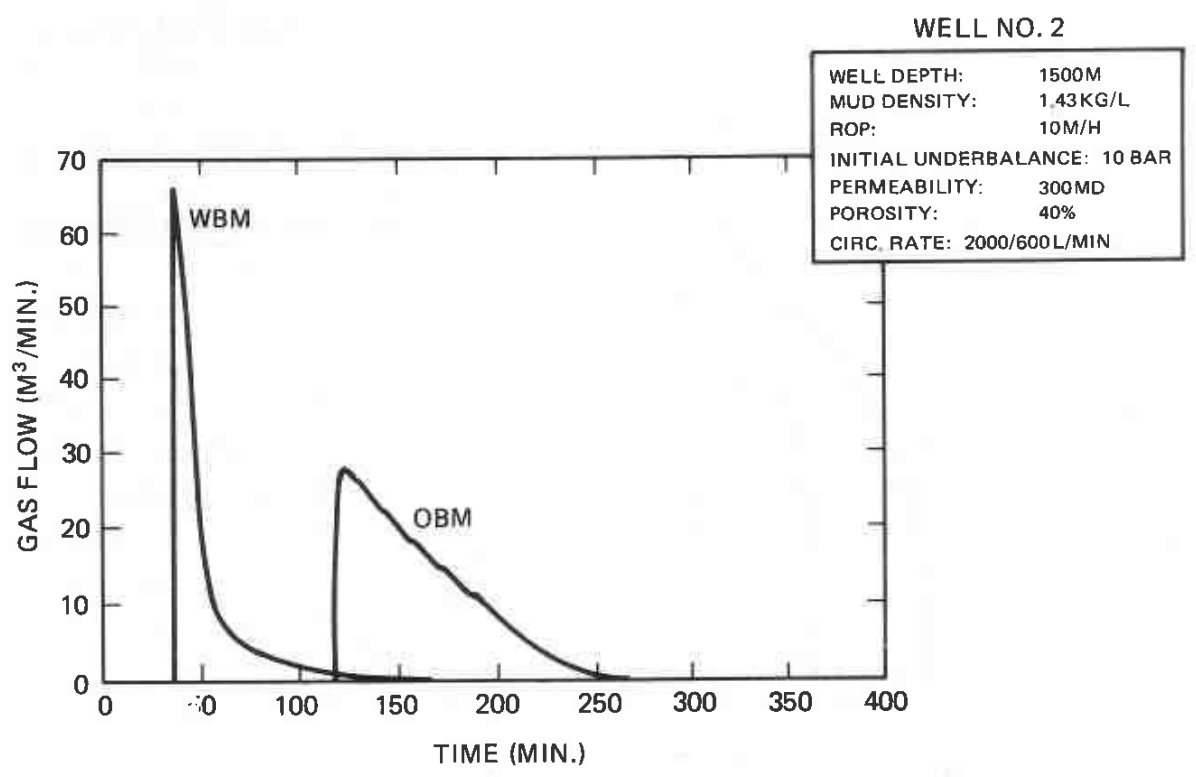

Figure 8. Gas flow rate through choke versus time for the kicks in the $1500 \mathrm{~m}$ deep well. 
(iii) This effect (decreasing pit gain) may be mistaken for lost circulation, and can lead to erronous actions by drilling crew.

(iv) Kicks in OBM give more gas to handle by surface equipment than kicks in WBM.

(v) It takes more time to empty the well of gas during kicks in OBM.

\section{Conclusions}

1. Gas kicks in oil-based drilling muds are so complicated that a numerical model should be used to describe the development of such kicks.

2. Such a model has been developed with success, and can be used for research purposes, field-related studies and training.

3. Gas kicks in OBM develop more slowly than gas kicks in WBM, but if the gas breaks out near the surface, the last phase of the kick will develop very rapidly.

4. Kicks in OBM are more difficult to detect than in WBM, and the well will contain more gas at detection point. Furthermore, the kick will be much closer to the surface when detected in OBM compared to WBM.

5. During the control phase of the kick the surface pit gain relationship is completely different in OBM from WBM, and even a decrease of pit gain prior to gas reaching the choke may happen in some cases. This must not be misinterpreted by the drilling crew.

\section{ACKNOWLEDGMENTS}

The authors are grateful to Statoil and Norsk Hydroa/s who have sponsored the development of the simulator. We are also indebted to our colleague Svein Halle who was very helpful during the simulations and preparations of the paper.

\section{REFERENCES}

DEPARTMENT OF ENERGY,(1987). Well control when drilling with oil-based mud. Report no. OTH 86260 , London.

EkranN, S. and Rommetveit, R. (1985). A Simulator for Gas Kicks in Oil-Based Drilling Muds. SPE paper 14182.

GARD, J. 1986). PVT-Measurements of Base Oils. Report PRC K-36/86, Rogaland Research Institute.

Halle, S. and Rommetveit, R. (1987). Pressure Control Training Course, Rogaland Research Institute.

Nickens, H. V. (1985). A Dynamic Computer Model of a Kicking Well. SPE paper 14183.

O’Bryan, P. L., Bourgoyne, JR, A. T., Monger, T. G. and KoPCso, D. P. (1988). An Experimental Study of Gas Solubility in Oil-Based Drilling Fluids. SPE Drilling Engineering, March.

Rommetveit, R. and BlyberG, A. (1986). Report PRC K-13/86, Rogaland Research Institute.

Rommetveit, R. and OlSEN, T. L. (1989). Gaskick Experiments in Oil-Based Drilling Muds in a Full-Scale, Inclined Research Well. SPE paper 19561, SPE Fall Conference 1989, San Antonio, Texas.

Swanson, B. W., Gilvary, B. and McEwan, F. (1988). SPE paper 18371, European Petroleum Conference, London. 